\title{
Novel Compact Multiband MIMO Antenna for Mobile Terminal
}

\author{
Cheng Yang, ${ }^{1}$ Yuan Yao, ${ }^{1}$ Junsheng Yu, ${ }^{1}$ and Xiaodong Chen ${ }^{2}$ \\ ${ }^{1}$ School of Electronic Engineering, Beijing University of Posts and Telecommunications, Xi Tu Cheng Road No.10, Haidian District, \\ Beijing 100876, China \\ ${ }^{2}$ School of Electronic Engineering and Computer Science, Queen Mary, University of London, Mile End Road, London E1 4NS, UK
}

Correspondence should be addressed to Cheng Yang, chengyang0565@126.com

Received 30 August 2011; Revised 9 October 2011; Accepted 18 October 2011

Academic Editor: Wenhua Chen

Copyright (C) 2012 Cheng Yang et al. This is an open access article distributed under the Creative Commons Attribution License, which permits unrestricted use, distribution, and reproduction in any medium, provided the original work is properly cited.

A novel compact MIMO antenna for personal digital assistant (PDA) and pad computer is proposed. The proposed antenna is composed by two multipatch monopole antennas which are placed $90 \circ$ apart for orthogonal radiation. To strengthen the isolation, a T-shaped ground branch with proper dimension is used to generate an additional coupling path to lower the mutual coupling (below -15 dB), especially at GSM850/900 band. The proposed MIMO antenna is fabricated and tested, both the simulated and the measured results are presented, and some parametric studies are also demonstrated. In addition, there are some advantages about the proposed antenna such as simple structure, easy fabrication, and low cost.

\section{Introduction}

With the rapid development of wireless communication, high data rate is required to improve the quality of information. However, the channel capacity of the conventional single-input, single-output (SISO) communication system is limited according to the Shannon's theorem. To solve this problem and reduce the signal fading in the rich scattering environment without any extra expenditure in power or spectrum, the multiple-input multiple-output (MIMO) communication system has been well developed in the past a few years $[1,2]$.

The printed monopole antennas are widely used in the MIMO communication systems for their advantages of low cost, easy fabrication, and good performance. To obtain the predicted high signal capacity, the mutual coupling between the antennas in the MIMO communication systems should be low enough, and the high isolation makes uncorrelated signals among the antennas [3]. Usually, low coupling can be obtained by separating the antennas at a distance of half a wavelength or more, but this is impractical in the mobile terminals, so a lot of methods have been studied to reduce the mutual coupling between the closely spaced monopole antennas.
Now many different MIMO antennas consisted of printed monopole antennas have been reported. In $[4,5]$, a quarter wavelength slot and its deformation are used to reduce the mutual coupling. In [6], parasitic elements are proposed for high isolation. In [7-9], different kinds of ground branches are reported to create an additional coupling path to cancel the original coupling. In [10-13], neutralization technique between antennas is proposed which can neutralize the current of two antennas. In $[14,15]$, decoupling networks based on lumped elements are adopted to strengthen the isolation between antennas. In [16], metamaterials are used to generate low correlation between antennas. Most of these MIMO antennas are worked at PCS, DCS, UMTS, or WLAN 2.4/5.2 bands with high isolation, but few of them can work at GSM 850/900 band with a strong isolation (below $-15 \mathrm{~dB}$ ) for mobile terminal.

In this paper, we present a novel compact multiband MIMO antenna with isolation enhancement using a $\mathrm{T}$ shaped ground branch for mobile terminal, such as PDA and pad computer. It is consisted of two symmetric printed monopole antennas and covers the GSM850/900, DCS, PCS, UMTS, and LTE2500 band with mutual coupling low enough (below $-15 \mathrm{~dB}$ ). Both the simulated and the measured results 
are represented, and the influence of the T-shaped ground branch on the isolation is analyzed.

\section{Antenna Design}

Figure 1 shows the geometry of the proposed multi-band MIMO antenna printed on a substrate of dimension $125 \times$ $100 \mathrm{~mm}^{2}$, which can represent the circuit board of the mobile terminal, such as PDA and pad computer. The substrate of the proposed antenna was chosen as low cost FR-4 material with a thickness of $0.8 \mathrm{~mm}, 4.4$ of dielectric constant, and 0.02 of loss tangent. Ansoft High-Frequency Structure Simulator (HFSS) was used to simulate and optimize the parameters of the proposed antenna.

The antenna is consisted of two orthogonal monopole antennas printed on the top layer of the substrate. The distance between the two monopole antennas is $36 \mathrm{~mm}$ which is only about $0.1 \lambda$ at $850 \mathrm{MHz}$. The radiated element of the monopole antenna is consisted of a rectangular patch with a folded slot inserted and a parasitic patch. The dimension of the rectangular patch is $33 \times 11 \mathrm{~mm}^{2}$. The folded slot divides the rectangular patch into two patches, and the inner patch (patch 2) is circled by the outer patch (patch 1). Patch 1 starts from the feed point and follows the folded slot to the open end of the slot while patch 2 starts from feed point to the end of inner patch circled by the folded slot, so the length of patch 1 is much longer than the length of patch 2 which makes patch 1 generate much lower operating band (GSM850/900) compared to the operating band (DCS, PCS, and UMTS) generated by patch 2 . To enlarge the high band, a parasitic patch (patch 3) is introduced which works at the LTE2500 band. By adjusting the lengths of the three patches properly, the desired operating bands can be obtained. The lengths of three patches are less than a quarter free wavelength of their own operating bands; this is mainly caused by the effect of the FR-4 substrate which decreases the resonant length of the radiating patches.

A $50 \mathrm{ohm}$ microstrip is used to feed the monopole antenna, which is also printed on the top layer of the substrate. Good impedance match can be obtained without any other match circuit.

The main ground plate of the MIMO antenna is about $85 \times 100 \mathrm{~mm}^{2}$ and there is a T-shaped branch extending from the main ground plate between the two monopole antennas. Both the T-shaped branch and the main ground plate are printed on the bottom layer of the substrate. The Tshaped branch is consisted of a vertical subbranch and a horizontal subbranch; the widths of the two subbranches are same to simplify the design of the proposed MIMO antenna. To obtain a high isolation, the dimensions of the two subbranches should be properly selected.

\section{Simulation and Experimental Results}

The proposed antenna shown in Figure 1 was fabricated and measured. The photo of the fabricated antenna is shown in Figure 2.

The simulated and experimental $S_{11}$ and $S_{21}$ parameters which were obtained using an Agilent 8753ES network

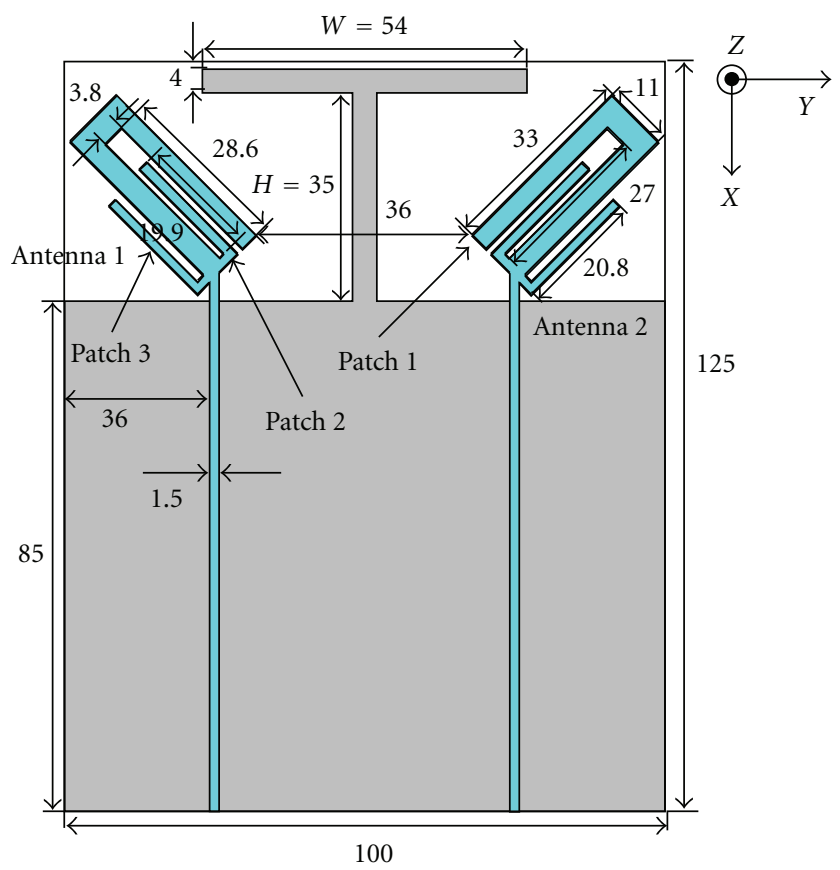

FIGURE 1: Geometry of the proposed multi-band MIMO antenna. (Unit: $\mathrm{mm}$ ).

analyzer are shown in Figures 3 and 4, respectively. Because of the symmetry of the MIMO antenna, there are only $S_{11}$ and $S_{21}$ shown. The measured results have some discrepancy compared to the simulated results which is probably caused by the manufactured and the measured tolerance. The $S_{11}$ bandwidth of the lowest band determined by VSWR $3: 1$ is from $0.81 \mathrm{GHz}$ to $1.02 \mathrm{GHz}$ which covers the GSM850 (824$894 \mathrm{MHz}) / 900(880-960 \mathrm{MHz})$ band, and the bandwidth of the middle band is from $1.45 \mathrm{GHz}$ to $2.2 \mathrm{GHz}$ and covers the DCS (1710-1880 MHz), PCS (1850-1990 MHz), and UMTS $(1920-2170 \mathrm{MHz})$ band. On the other hand, the bandwidth of high band caused by the parasitic patch is from $2.39 \mathrm{GHz}$ to $2.8 \mathrm{GHz}$ and satisfies the requirement of LTE2500 (2500$2690 \mathrm{MHz}$ ) band. The measured $S_{21}$ parameter in all of these bands is bellow $-15 \mathrm{~dB}$ and this value is low enough for mobile terminal. From Figure 4 it can be seen that the isolation between two ports is worse in the lower band than in the higher band, this is mainly because the normalized distance by wavelength between the ports is shorter in the lower band.

The simulated 3D radiation patterns of antenna 1 and antenna 2 at $0.9 \mathrm{GHz}, 1.9 \mathrm{GHz}$, and $2.5 \mathrm{GHz}$ are shown in Figures 5, 6 and 7, respectively. From the results it can be seen that the radiation patterns of the two antennas are orthogonal to each other, so the dual-element MIMO antenna shows good pattern diversity characteristic to overcome the multipath fading and enhance the system performance. The simulated gain in all the bands is high than $0 \mathrm{~dB}$, and the value of the gain has a little difference between antenna 1 and antenna 2. The far field measurements were carried out in an anechoic chamber. When one antenna was measured, the other one was terminated by a $50 \mathrm{ohm}$ load. Figures 8,9 and 10 show the radiation patterns in the yoz plane and in the $x o z$ 


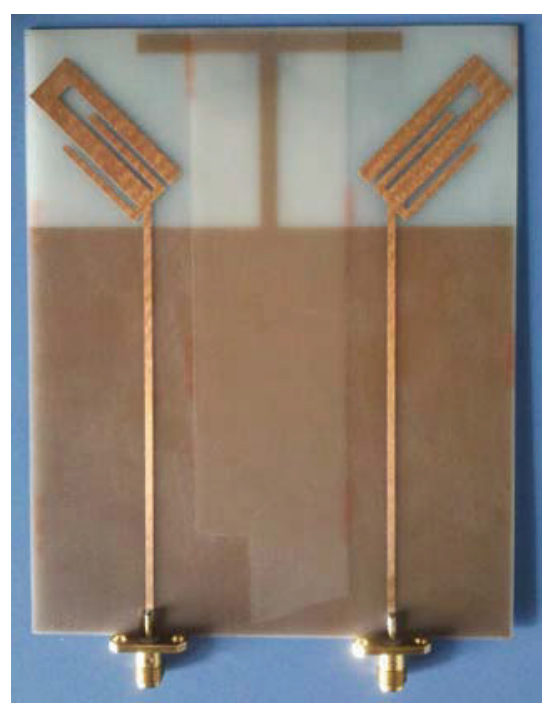

(a)

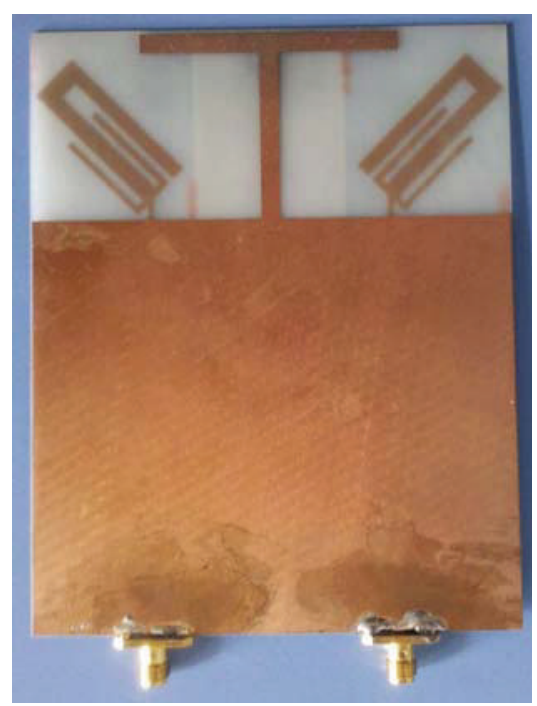

(b)

Figure 2: Photographs of the fabricated MIMO antenna.

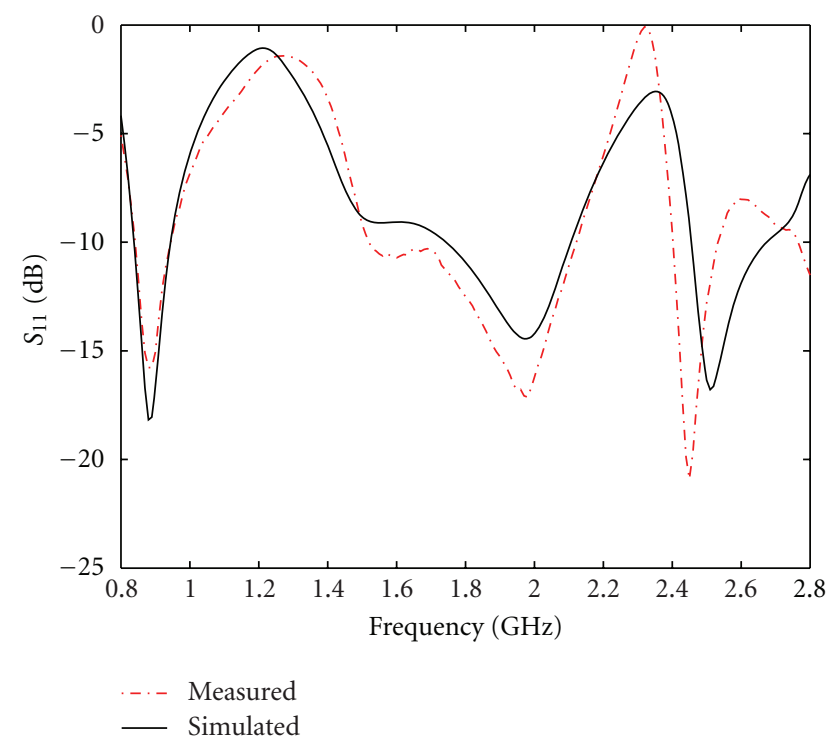

FIgURE 3: Measured and simulated $S_{11}$ of the proposed antenna.

plane at $0.9,1.9$, and $2.5 \mathrm{GHz}$, respectively. The results also show that the radiation fields of the antenna 1 and antenna 2 are orthogonal. The discrepancy of the patterns between the two antennas is mainly caused by the manufactured and the measured aberration.

Usually, the envelope correlation coefficient is an important parameter to evaluate the diversity characteristic of a multi-antenna system [17]. Low envelope correlation coefficient means high diversity gain. Generally speaking, this value should be less than 0.5 to get a good characteristic of diversity for the mobile terminal application [18]. The envelope

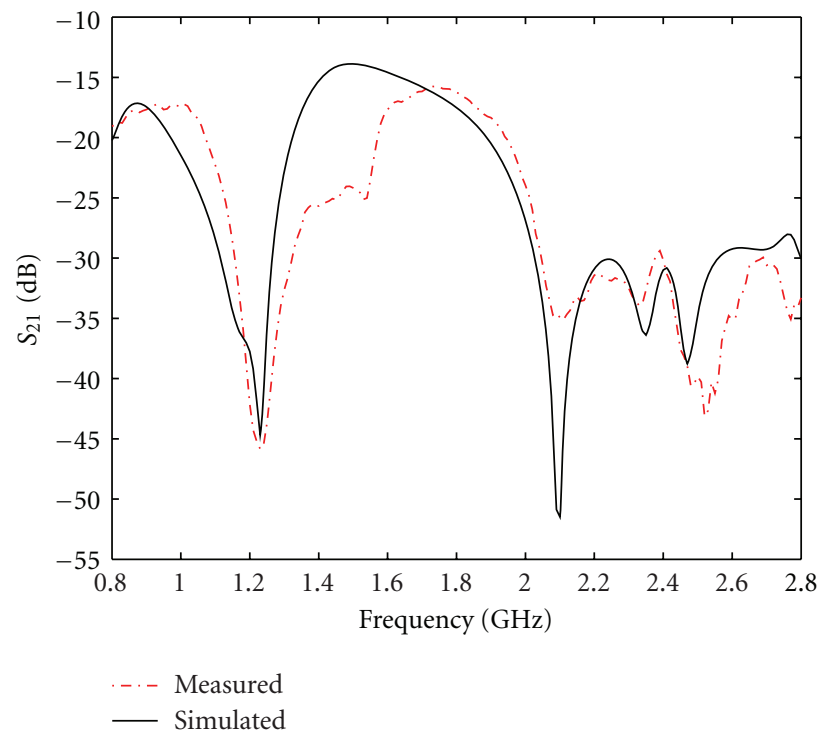

Figure 4: Measured and simulated $S_{21}$ of the proposed antenna.

correlation coefficient computed from the measured $S$ parameters is shown as follows [19]:

$$
\rho_{e}=\frac{\left|S_{11}^{*} S_{12}+S_{21}^{*} S_{22}\right|^{2}}{\left[1-\left(\left|S_{11}\right|^{2}+\left|S_{21}\right|^{2}\right)\right]\left[1-\left(\left|S_{22}\right|^{2}\left|S_{12}\right|^{2}\right)\right]} .
$$

From the measured $S$ parameters, the envelope correlation coefficient was calculated, which is shown in Figure 11. In the GSM850/900 band, the maximum coefficient is 0.005 , on the other hand, the maximum coefficients in the middle bands and the LTE2500 band are 0.0127 and 0.001 , respectively. The obtained envelope correlation coefficient shows that the proposed antenna is hopeful for MIMO application. 

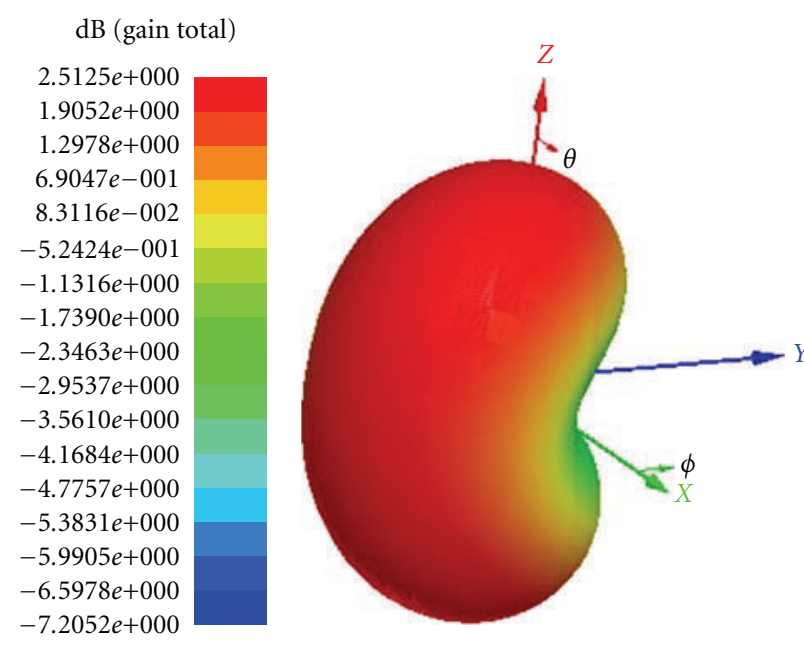

(a)
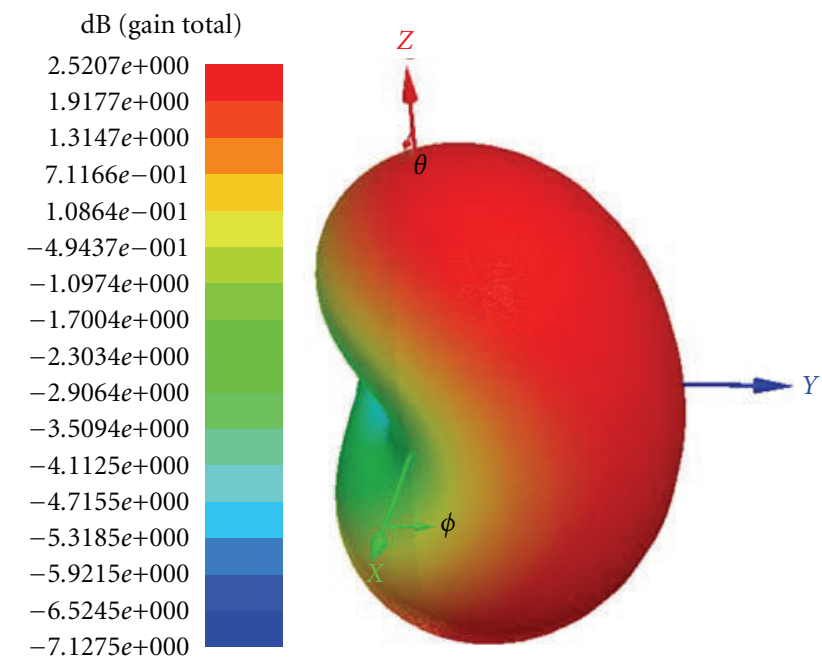

(b)

Figure 5: Simulated 3D radiation pattern at $0.9 \mathrm{GHz}$. (a) Antenna 1 excited. (b) Antenna 2 excited.
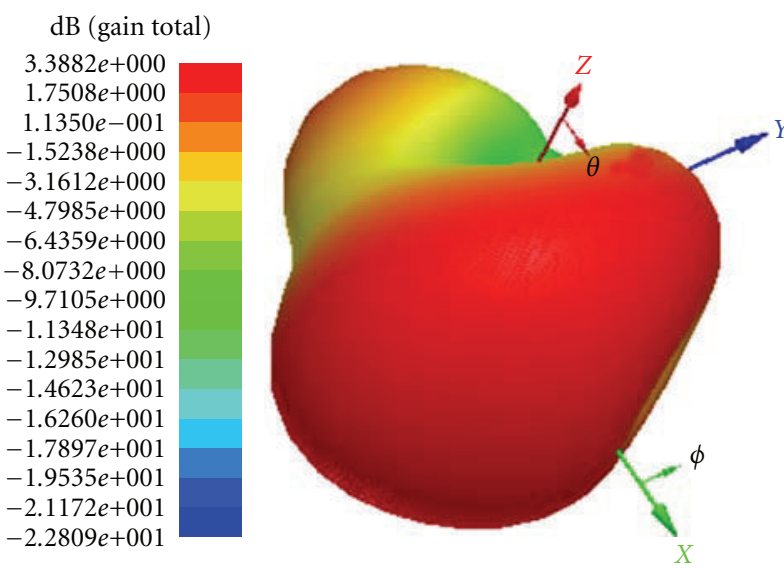

(a)
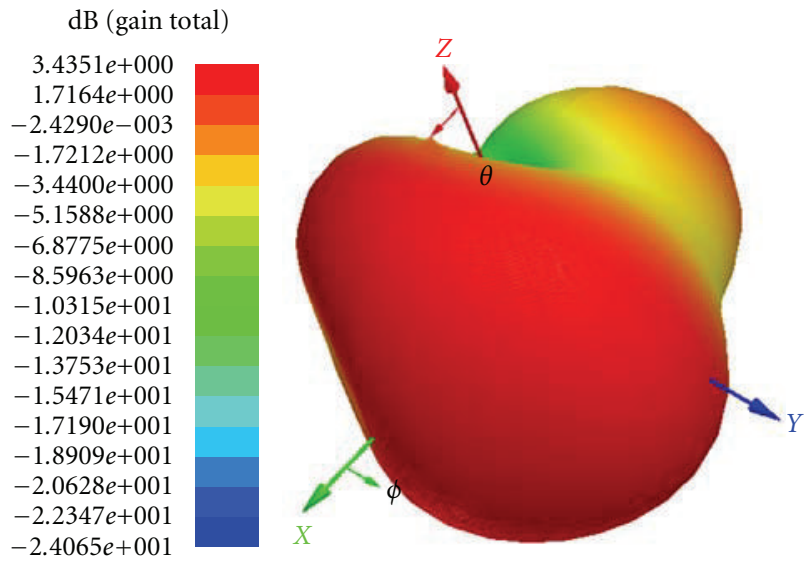

(b)

Figure 6: Simulated 3D radiation pattern at 1.9 GHz. (a) Antenna 1 excited. (b) Antenna 2 excited.
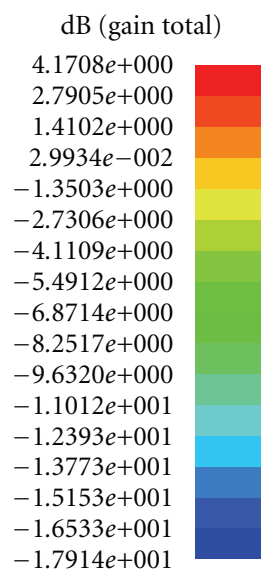

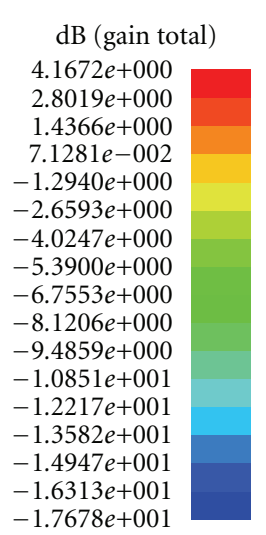

$-1.7678 e+001$

(a)

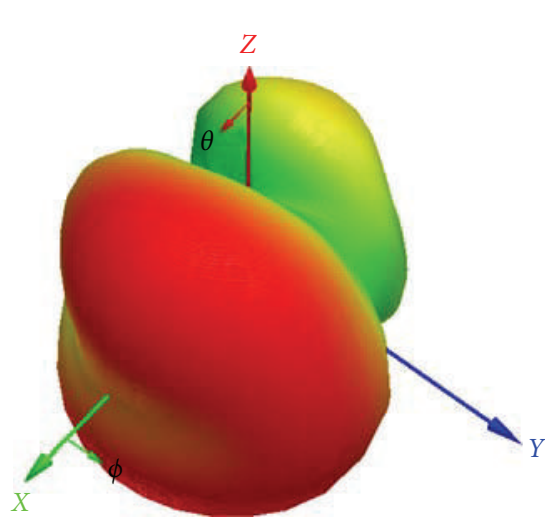

(b)

Figure 7: Simulated 3D radiation pattern at 2.5 GHz. (a) Antenna 1 excited. (b) Antenna 2 excited. 


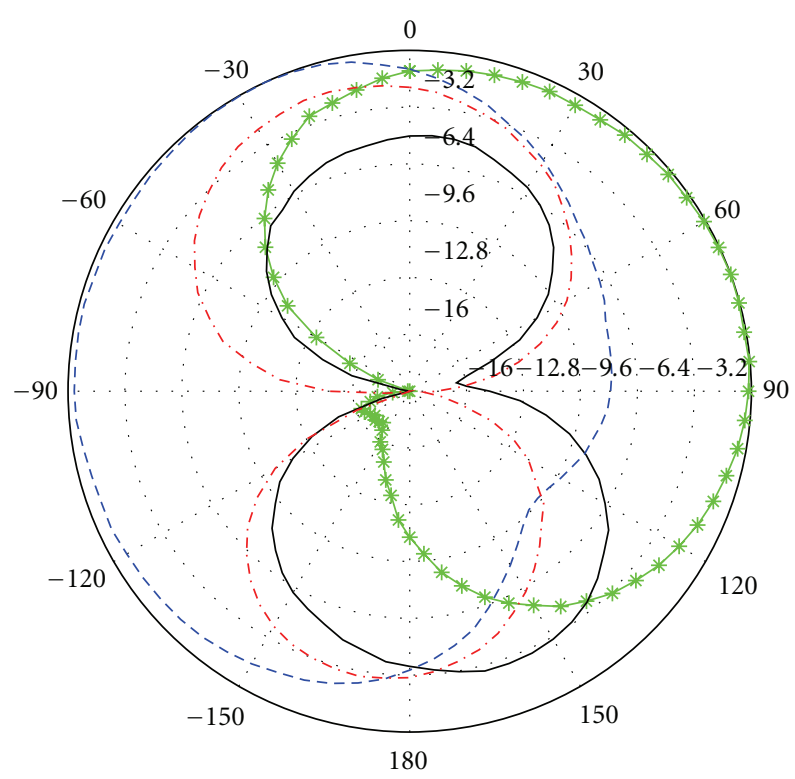

Antenna $1 \mathrm{E}$

-. $\theta$

$---\phi$
Antenna $2 \mathrm{E}$

- $\theta$

(a)

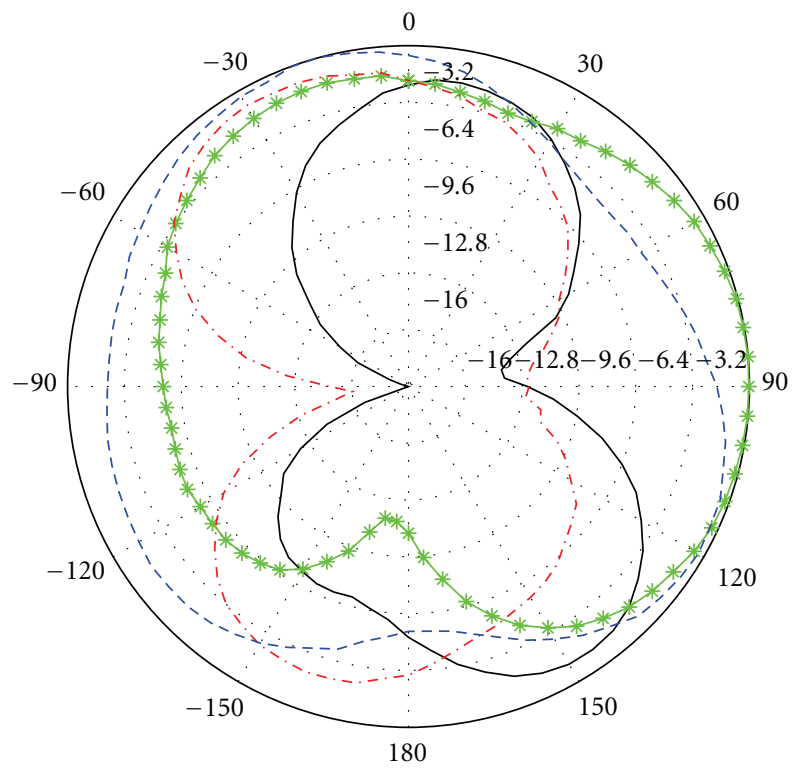

Antenna $1 \mathrm{E}$

-.. $\theta$

$---\phi$

Antenna 2 E

- $\begin{array}{r}-\theta * * \\ \phi\end{array}$

(b)

Figure 8: Measured radiation pattern at $0.9 \mathrm{GHz}$. (a) The yoz plane. (b) The xoz plane.

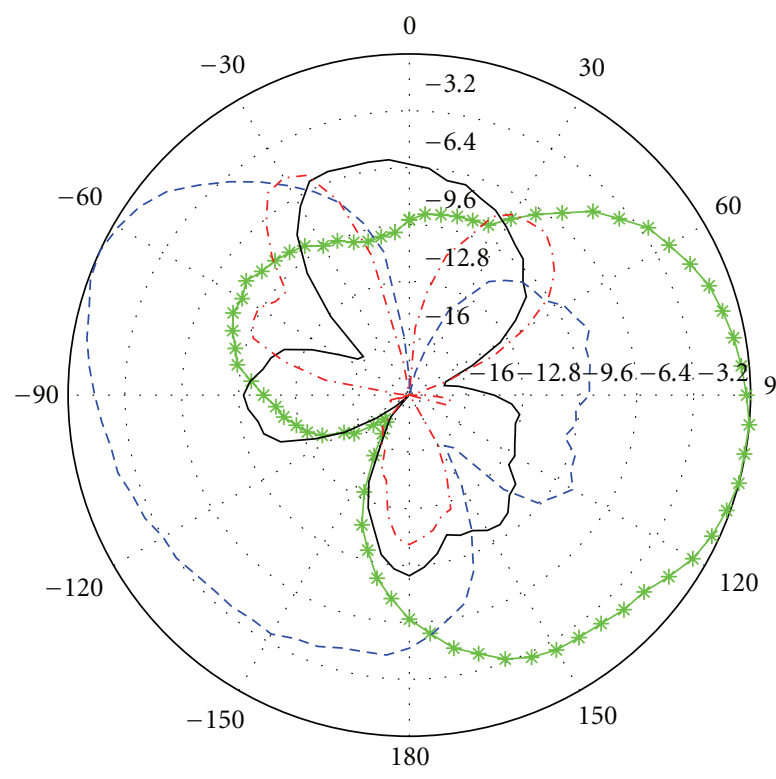

Antenna $1 \mathrm{E}$

$\cdots-\theta$

$---\phi$
Antenna $2 \mathrm{E}$

- $\theta$

(a)

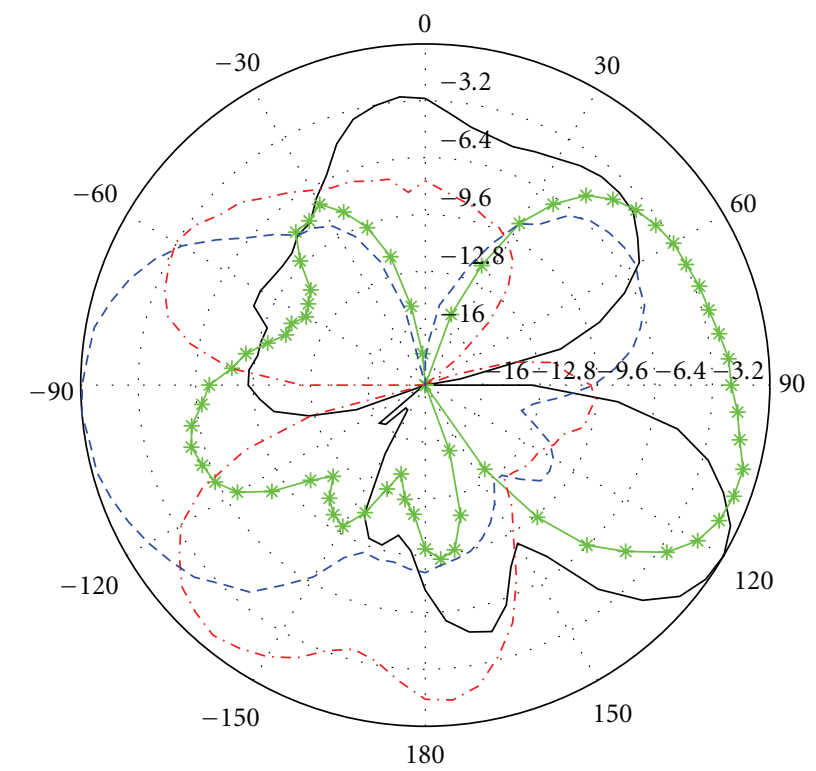

Antenna $1 \mathrm{E}$

$\cdots \theta$

$---\phi$

(b)

FIgURE 9: Measured radiation pattern at $1.9 \mathrm{GHz}$. (a) The yoz plane. (b) The $x o z$ plane. 


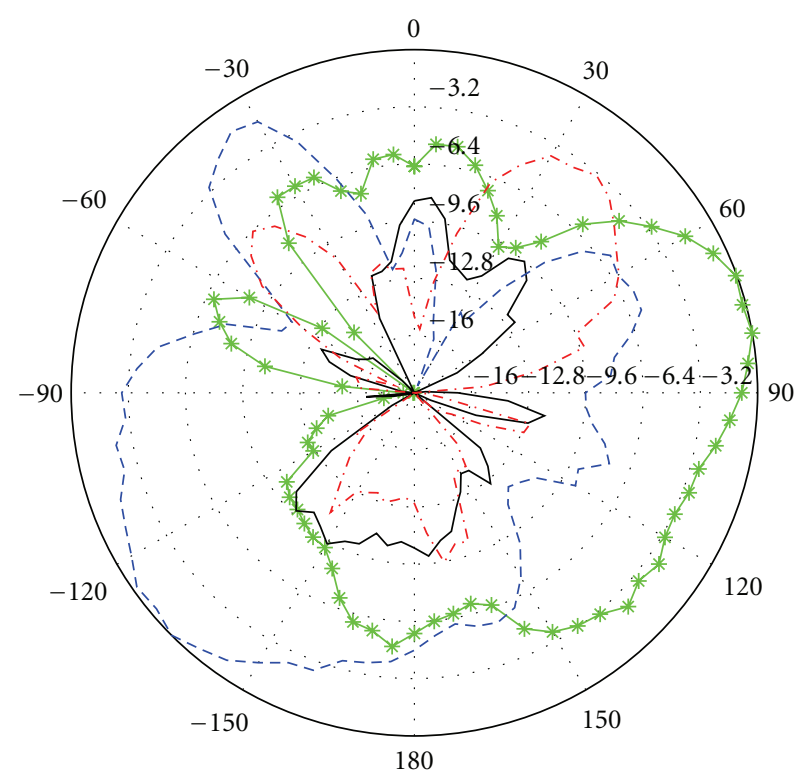

Antenna $1 \mathrm{E}$

$\cdot-\cdot \theta$

$---\phi$
Antenna $2 \mathrm{E}$

$-\theta$

(a)

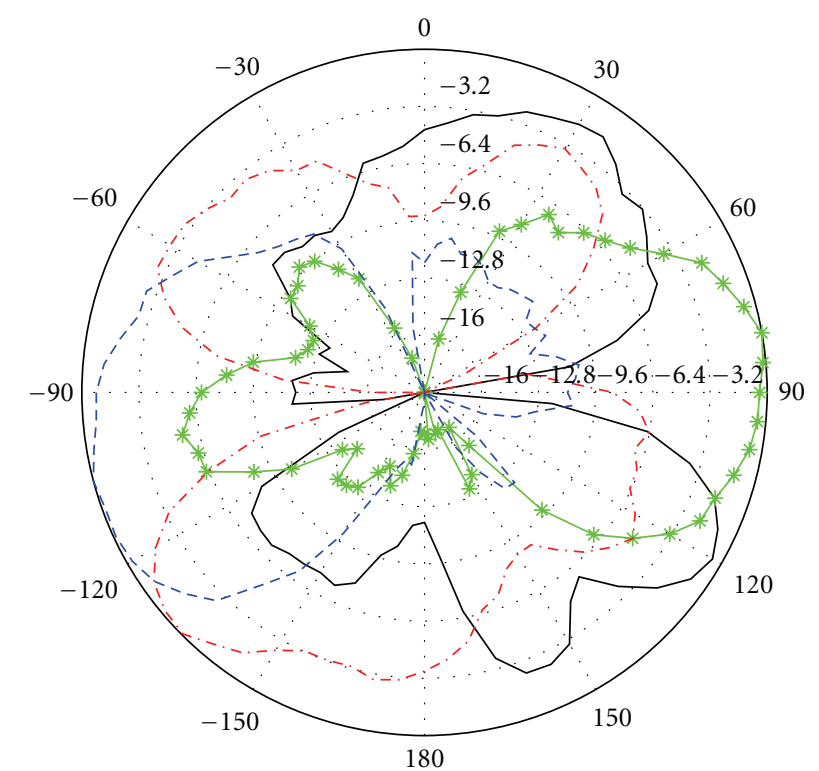

Antenna $1 \mathrm{E}$

$-\cdot \theta$

$---\phi$

Antenna $2 \mathrm{E}$

$-\theta$

(b)

Figure 10: Measured radiation pattern at $2.5 \mathrm{GHz}$. (a) The yoz plane. (b) The xoz plane.

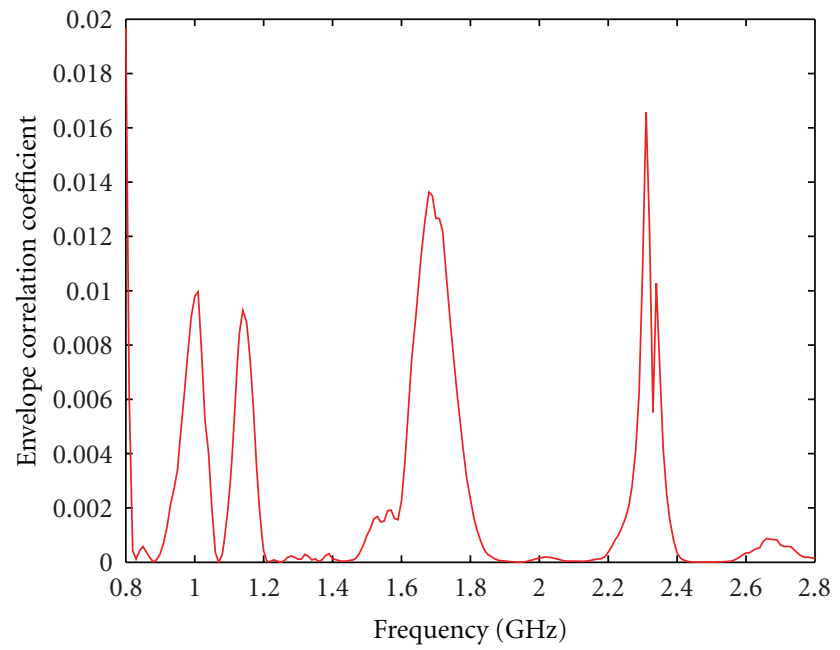

FIGURE 11: Envelope correlation coefficient computed from the measured $S$ parameters.

\section{Parametric Studies on Decoupling Element}

To further demonstrate the operation of the decoupling element, we have made some parametric studies on the Tshaped ground branch and show the current distribution on the MOMO antenna.

Figure 12 shows the simulated results of the $S_{21}$ parameter of the MIMO antenna with the T-shaped branch or not.

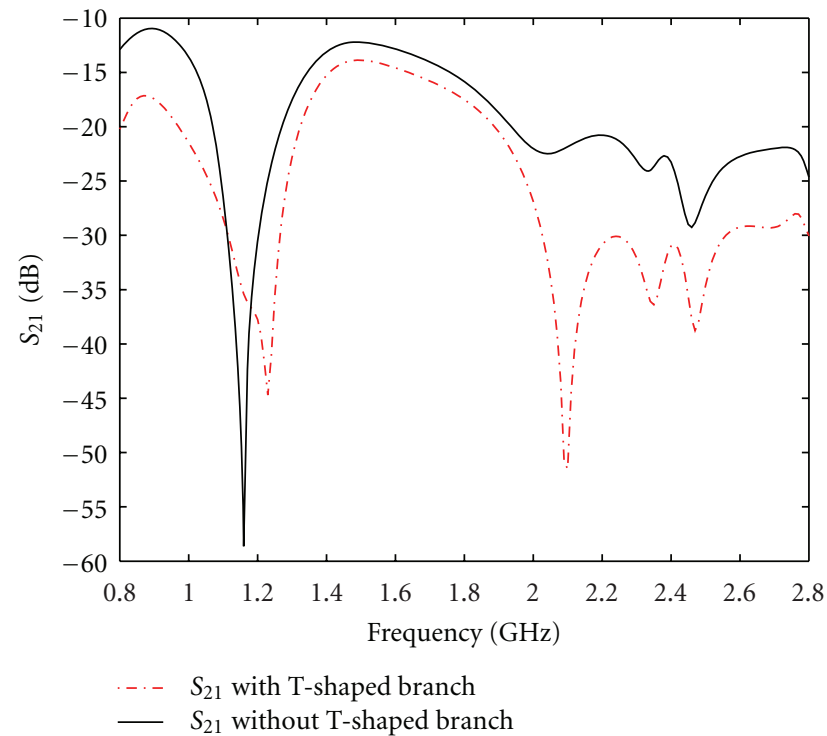

FIGURE 12: Simulated results of $S_{21}$ of the antenna with the T-shaped branch or not.

From the result it can be seen that the mutual coupling between antenna 1 and antenna 2 is greatly improved. With the T-shaped branch, the maximum $S_{21}$ at the GSM 850/900 band is from $-11 \mathrm{~dB}$ to $-17 \mathrm{~dB}$, at the middle band is from $-14 \mathrm{~dB}$ to $-16 \mathrm{~dB}$, and at the LTE2500 band is from $-22 \mathrm{~dB}$ to $-29 \mathrm{~dB}$. With the T-shaped ground branch, the isolation 

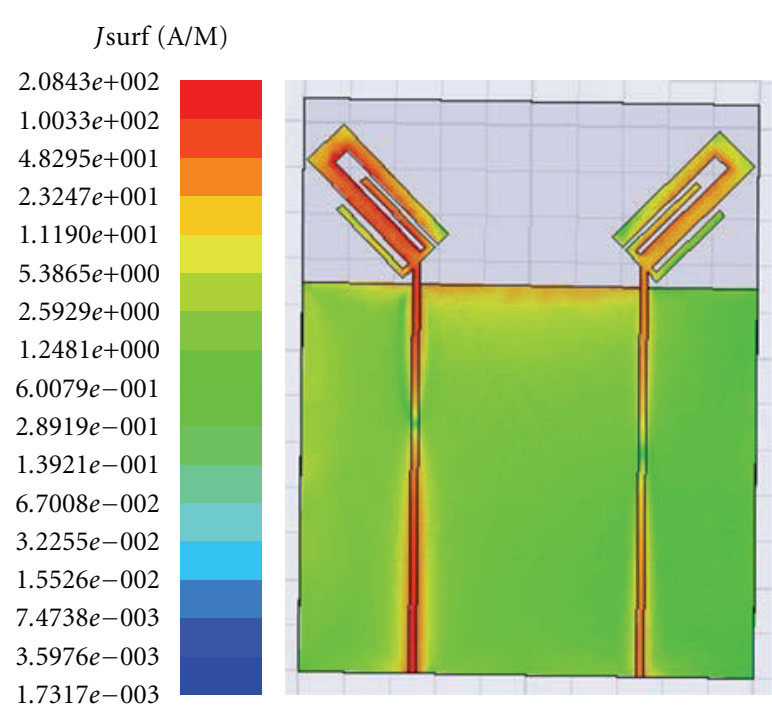

(a)
$J \operatorname{surf}(\mathrm{A} / \mathrm{M})$

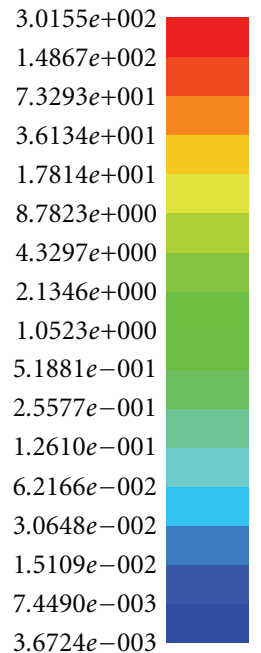

$3.6724 e-003$

(b)

FIgURE 13: Simulated surface current distribution at $0.9 \mathrm{GHz}$. (a) Without the T-shaped branch. (b) With the T-shaped branch.

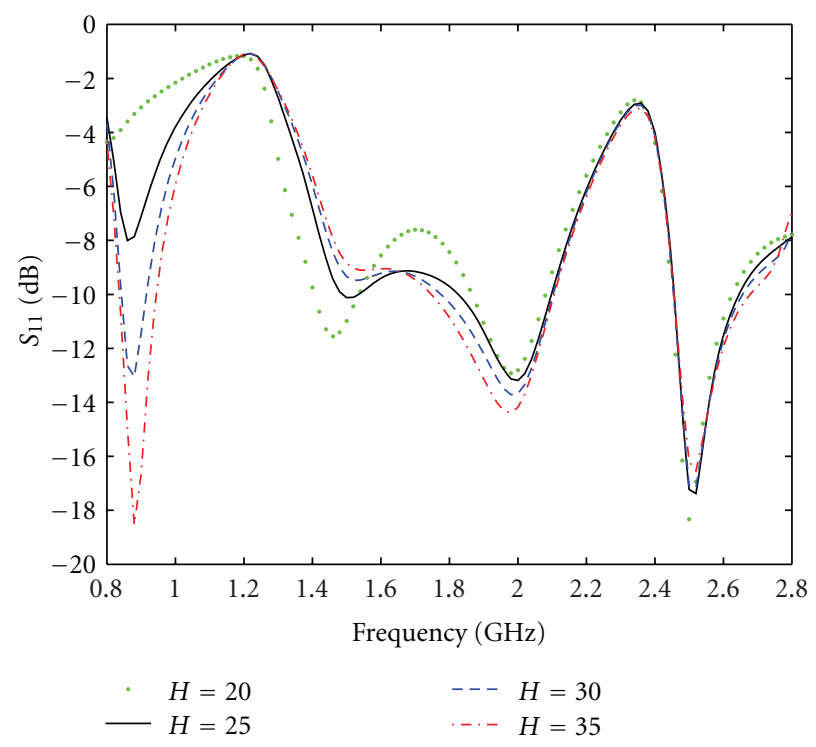

(a)

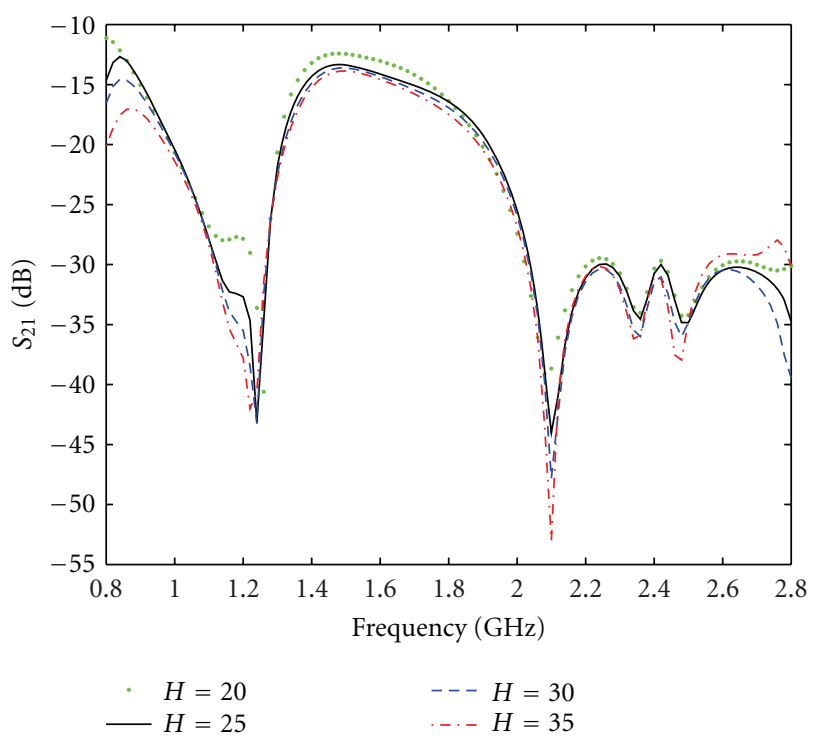

(b)

FIGURE 14: Simulated results of $S$ parameters when parameter $H$ varied (Unit: mm). (a) $S_{11}$. (b) $S_{21}$.

between the two monopole antennas is strong enough for mobile terminal.

To explain how the T-shaped ground patch reduces the mutual coupling, the surface current distribution is shown. Figure 13(a) shows the surface current distribution on the entire element without the T-shaped branch while antenna 1 is excited. In this case, the surface current induced on the antenna 2 is strong, so the mutual coupling is high. At Figure 13(b), when the T-shaped branch is added to the ground plate, the induced surface current on the antenna 2 is much weaker where the antenna 1 is excited as before, so the mutual coupling is much lower. The reason is that the antenna 1 induces coupling current on the T-shaped branch and the antenna 2 , respectively, and the T-shaped branch also induces coupling current on the antenna 2 where the two induced coupling currents on the antenna 2 are reverse, so the isolation is strengthened.

Figure 14 shows the effect of various values of the parameter $H$ which is shown in Figure 1 on the simulated $S_{11}$ and $S_{21}$ parameters for the proposed MIMO antenna while other parameters are fixed. From the picture it can be seen that the impedance matching is improved and the isolation between the two antennas is strengthened when the value of the parameter $H$ increases, especially at the lower bands. Besides 


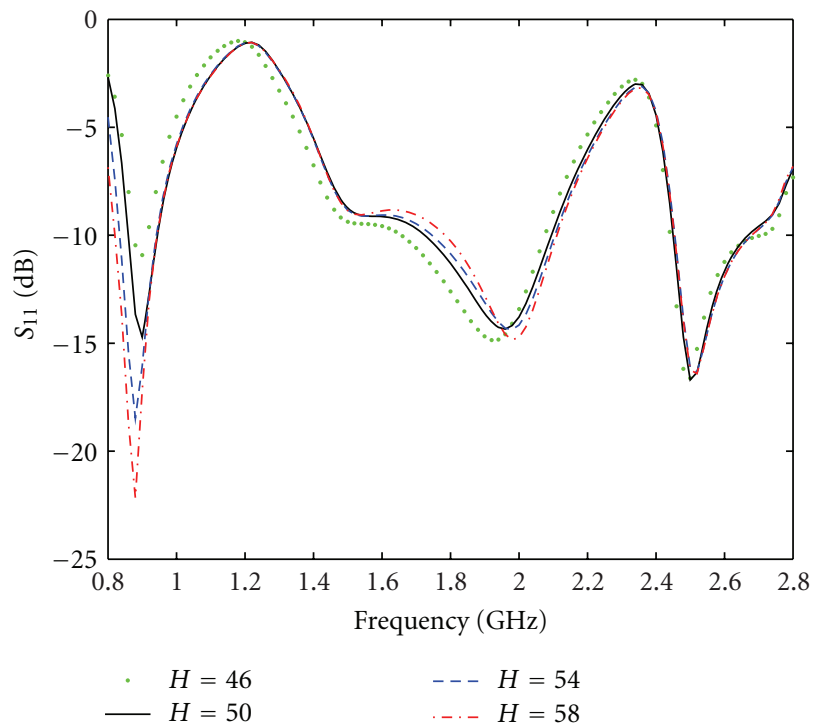

(a)

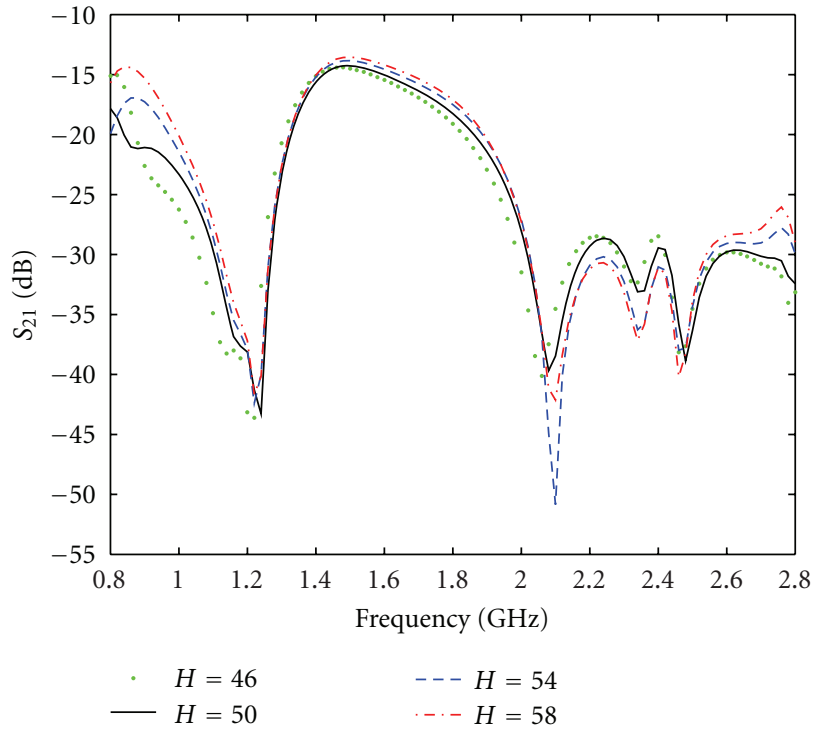

(b)

FIgURE 15: Simulated results of $S$ parameters when parameter $W$ varied (Unit: mm). (a) $S_{11}$. (b) $S_{21}$.

these, the resonant frequencies are not changed much when the parameter $H$ varies. So the parameter $H=35 \mathrm{~mm}$ was chosen for the proposed antenna.

The effect of various values of the parameter $W$ on the simulated $S_{11}$ and $S_{21}$ parameters is shown in Figure 15; other parameters are fixed. From the results we can see that the impedance matching is improved when the value of the parameter $W$ increases. For the isolation, there exists an optimal value of $W$. In this study, when $W=50 \mathrm{~mm}$, the mutual coupling between the antennas is lowest in the desired bands. But in this situation, the $S_{11}$ band at GSM850 band is not wide enough, so a trade-off between the bandwidth and the isolation should be made, and we chose $W=$ $54 \mathrm{~mm}$ for our design.

\section{Conclusion}

In this paper, a novel compact MIMO antenna for GSM850/ 900, PCS, DCS, UMTS, and LTE2500 bands has been proposed and studied. The MIMO antenna is consisted of two orthogonal monopole antennas and the monopole antenna is formed by three patches with different lengths. To strength the isolation between the two ports, a T-shaped ground branch between the two monopoles is adopted. Furthermore, the decoupling mechanism of the T-shaped ground branch is proposed, and some parametric studies on the branch have been made to make a good impedance matching and a high isolation. The prototypes of the proposed MIMO antenna have been successfully implemented and good antenna performances have been observed. The return loss $\left(S_{11}\right)$ is below $-6 \mathrm{~dB}$ and the isolation characteristic $\left(S_{21}\right)$ is less than $-15 \mathrm{~dB}$ in all the desired frequency bands, especially at GSM850/900 band. Both the simulated and the measured patterns are given and the orthogonal radiation characteristic is observed. Moreover, the envelope correlation coefficient of this MIMO antenna is far less than 0.5 which leads to a good diversity characteristic to overcome the multipath fading. The design is simple and constructed with a low cost which is promising for mobile terminal such as PDA and pad computer.

\section{Acknowledgment}

The authors wish to acknowledge the support of the Fundamental Research Funds for the Central Universities.

\section{References}

[1] R. D. Murch and K. Ben Letaief, "Antenna systems for broadband wireless access," IEEE Communications Magazine, vol. 40, no. 4, pp. 76-83, 2002.

[2] J. W. Wallace, M. A. Jensen, A. L. Swindlehurst, and B. D. Jeffs, "Experimental characterization of the MIMO wireless channel: data acquisition and analysis," IEEE Transactions on Wireless Communications, vol. 2, no. 2, pp. 335-343, 2003.

[3] T. Svantesson and A. Ranheim, "Mutual coupling effects on the capacity of multielement antenna systems," in Proceedings of the IEEE Interntional Conference on Acoustics, Speech, and Signal Processing (ICASSP '01), pp. 2485-2488, May 2001.

[4] K. J. Kim and K. H. Ahn, "The high isolation dual-band inverted $\mathrm{F}$ antenna diversity system with the small N-section resonators on the ground plane," Microwave and Optical Technology Letters, vol. 49, no. 3, pp. 731-734, 2007.

[5] G. A. Mavridis, J. N. Sahalos, and M. T. Chryssomallis, "Spatial diversity two-branch antenna for wireless devices," Electronics Letters, vol. 42, no. 5, pp. 266-268, 2006.

[6] Z. Li, Z. Du, and K. Gong, "A dual-slot diversity antenna with isolation enhancement using parasitic elements for mobile handsets," in Proceedings of the Asia Pacific Microwave Conference (APMC '09), pp. 1821-1824, December 2009. 
[7] A. C. K. Mak, C. R. Rowell, and R. D. Murch, "Isolation enhancement between two closely packed antennas," IEEE Transactions on Antennas and Propagation, vol. 56, no. 11, pp. 3411-3419, 2008.

[8] Y. Ding, Z. Wu, K. Gong, and Z. Feng, "A novel dual-band printed diversity antenna for mobile terminals," IEEE Transactions on Antennas and Propagation, vol. 55, no. 7, pp. 20882096, 2007.

[9] H. Chung, Y. Jang, and J. Choi, "Design of a multiband internal antenna for mobile application," in Proceedings of the IEEE International Symposium on Antennas and Propagation and USNC/URSI National Radio Science Meeting (APSURSI '09), pp. 1-4, June 2009.

[10] Q. Luo, J. R. Pereira, and H. M. Salgado, "Reconfigurable dualband C-shaped monopole antenna array with high isolation," Electronics Letters, vol. 46, no. 13, pp. 888-889, 2010.

[11] Q. Luo, H. M. Salgado, and J. R. Pereira, "Printed C-shaped monopole antenna array with high isolation for MIMO applications," in Proceedings of the IEEE International Symposium on Antennas and Propagation and CNC-USNC/URSI Radio Science Meeting, pp. 1-4, July 2010.

[12] I. Dioum, A. Diallo, C. Luxey, and S. M. Farsi, "Dual-band monopole MIMO antennas for LTE mobile phones," in Proceedings of the ICECom Conference, pp. 1-4, 2010.

[13] G. Park, M. Kim, T. Yang, J. Byun, and A. S. Kim, "The compact quad-band mobile handset antenna for the LTE700 MIMO application," in Proceedings of the IEEE International Symposium on Antennas and Propagation and USNC/URSI National Radio Science Meeting (APSURSI '09), pp. 1-4, June 2009.

[14] S. C. Chen, Y. S. Wang, and S. J. Chung, "A decoupling technique for increasing the port isolation between two strongly coupled antennas," IEEE Transactions on Antennas and Propagation, vol. 56, no. 12, pp. 3650-3658, 2008.

[15] C. Y. Lui, Y. S. Wang, and S. J. Chung, "Two nearby dualband antennas with high port isolation," in Proceedings of the IEEE International Symposium on Antennas and Propagation and USNC/URSI National Radio Science Meeting (APSURSI '08), pp. 1-4, July 2008.

[16] C. C. Hsu, K. H. Lin, H. L. Su, H. H. Lin, and C. Y. Wu, "Design of MIMO antennas with strong isolation for portable applications," in Proceedings of the IEEE International Symposium on Antennas and Propagation and USNC/URSI National Radio Science Meeting (APSURSI '09), pp. 1-4, June 2009.

[17] S. C. K. Ko and R. D. Murch, "Compact integrated diversity antenna for wireless communications," IEEE Transactions on Antennas and Propagation, vol. 49, no. 6, pp. 954-960, 2001.

[18] R. G. Vaughan and J. B. Andersen, "Antenna diversity in mobile communications," IEEE Transactions on Vehicular Technology, vol. 36, no. 4, pp. 149-172, 1987.

[19] S. Blanch, J. Romeu, and I. Corbella, "Exact representation of antenna system diversity performance from input parameter description," Electronics Letters, vol. 39, no. 9, pp. 705-707, 2003. 

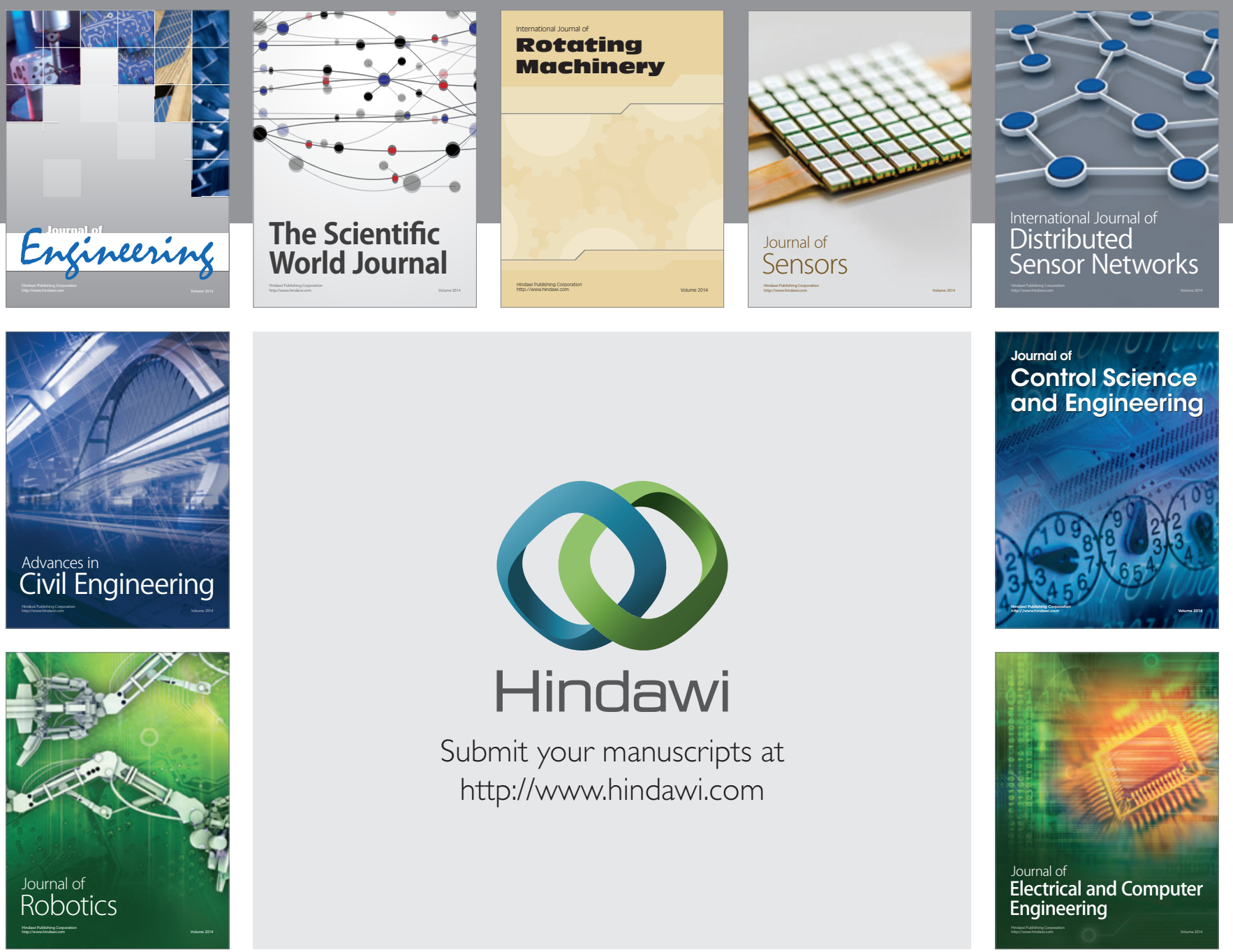

Submit your manuscripts at

http://www.hindawi.com
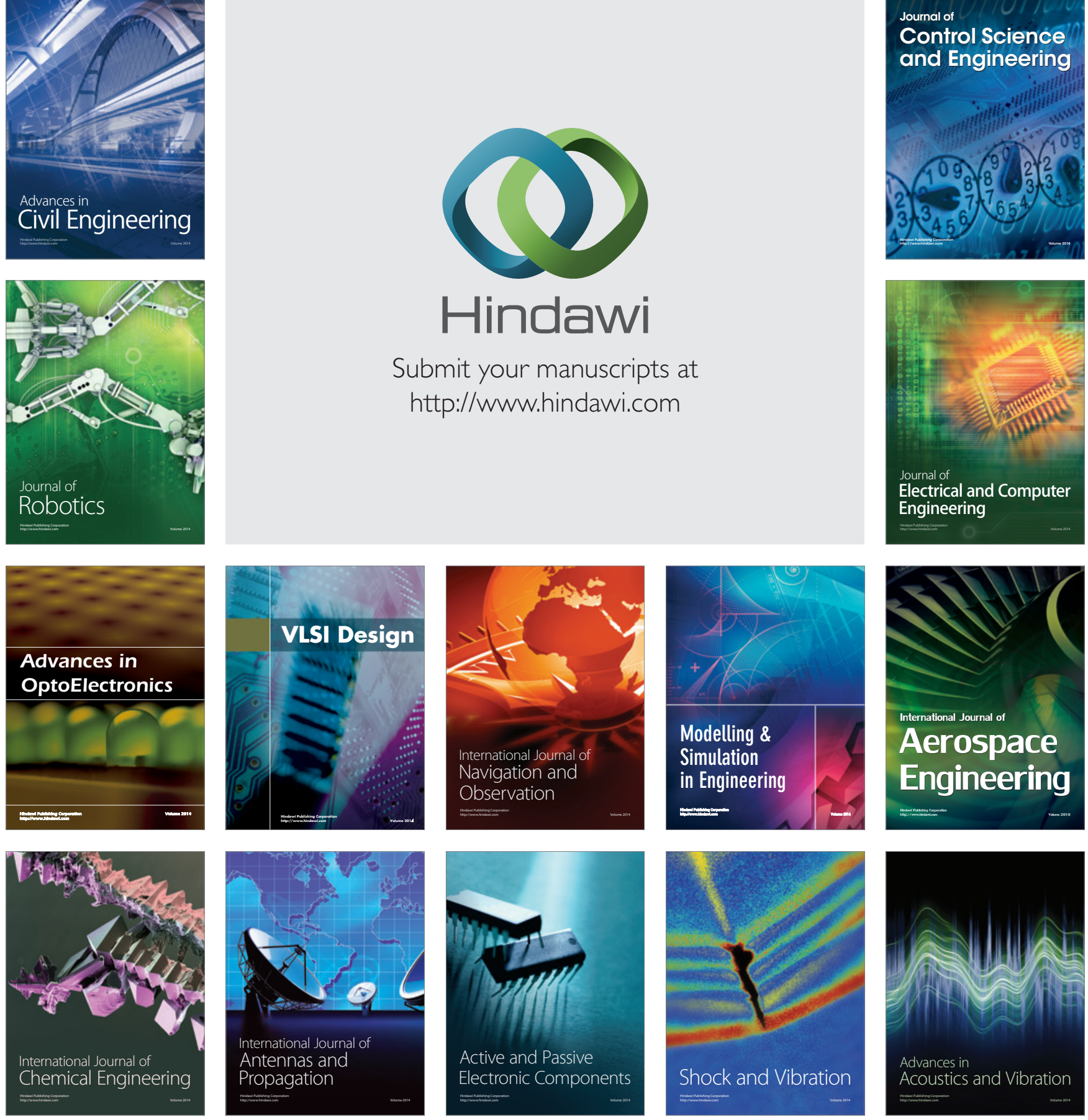